\title{
NADPH Oxidase: The Culprit in Impaired Autophagy and Lysosomal Biogenesis?
}

\author{
Rituraj Pal* \\ Department of Molecular Physiology and Biophysics, Baylor College of Medicine, Houston, Texas, USA
}

\begin{abstract}
Received: June 26, 2014; Accepted: July 10, 2014; Published: July 17, 2014
*Corresponding author: Rituraj Pal, Department of Molecular Physiology and Biophysics, Baylor College of Medicine, Houston, Texas, USA, E-mail: rpal@bcm.edu
\end{abstract}

Autophagy is a dynamic cellular degradative pathway involved in the degradation of organelles, protein aggregates and other cellular constituents. While the ubiquitin proteasome system delivers degradation of smaller protein aggregates, autophagy maintains cellular housekeeping machinery by eliminating larger damaged constituents that could otherwise become toxic [1]. Although autophagy is often referred to as degradative, it is more precise to describe it as a recycling pathway involved in generating metabolites by fusing with lysosomes [2]. Autophagy has been characterized by two events, autophagosome formation and then fusion with the lysosomes to form autolysosomes in mammalian cells [3]. Therefore, both autophagosome formation and lysosomal biogenesis are imperative to maintain the homeostasis of autophagic machinery.

Recent evidence supports both, the damage of the lysosomal membrane and protection of the lysosomal compartment in response to oxidative stress. While oxidative stress-induced breakdown of lysosomes are intensively reported, these two juxtaposed fundamentals need to be further studied [4]. Since lysosomes are the key organelles in autolysosome-mediated degradation of toxic aggregates, understanding the interface between source of oxidative stress and its mechanism of action on impaired autophagy has recently gained immense importance in basic as well as translational aspects of research.

NADPH (Nicotinamide Adenine Dinucleotide Phosphate) Oxidase has been persistently reported as a major source of oxidative stress in several pathologies. NADPH Oxidase enzyme complex comprises the membrane-bound catalytic gp91 ${ }^{\text {phox }}$ (Nox2, homologes are Nox1, Nox3, Nox4 and Nox5) and the p22 $2^{\text {phox }}$ subunits, as well as cytosolic subunits including $\mathrm{p} 47^{\text {phox }}, \mathrm{p} 67^{\text {phox }}$ and Rac1. In cellular homeostasis, physiological concentration of ROS generated by NADPH oxidases acts as an effector signaling molecule for cell growth, migration and several other physiological responses [5]. Over activation of NADPH Oxidase induces excess ROS and subsequent oxidative stress, which has been implicated with various pathological disorders. However the association between NADPH Oxidase-induced oxidative stress and impaired autophagolysosome formation is poorly understood.
Recent evidence supports Src kinase-dependent activation of $\mathrm{p} 47^{\text {phox }}$ stimulates the translocation of $\mathrm{p} 47^{\text {phox }}$ from cytosol to membrane, which then form complex with Nox2 and activates the enzyme complex [6]. Our recent studies strongly corroborate the fact that NADPH Oxidase-induced ROS is involved in multiple pathological conditions. Activation of Src induces activation of mammalian target of rapamycin (mTOR) via activating PI3K/ Akt pathway and results in blockade in autophagy [7]. We have strong evidences that inhibition of NADPH Oxidase or Src kinase induces autophagy in multiple disease models by suppressing Src/PI3K/Akt/mTOR signaling pathway [8]. Transcription factorEB (TFEB) has recently been reported as a master regulator of lysosomal biogenesis by regulating the genes responsible for lysosomal expression and regulation network. Phosphorylation in TFEB by mitogen-activated protein kinases (ERK1/2) controls cytosolic localization of TFEB. Inhibition of ERK1/2 results in translocation of TFEB to nuclear region, an event promotes lysosomal biogenesis [9]. Oxidative stress-induced activation of ERK1/2 has been intensively reported in last decade. Although the specific source of the oxidative stress (involved in activation of ERK1/2) and mechanism of action is not clearly understood, together these studies suggest a possible involvement of NADPH Oxidase-dependent oxidative stress in phosphorylation of TFEB through ERK1/2-activation, prevents translocation of TFEB from cytosol to nucleus. Therefore, this could strongly be a possible mechanism of oxidative stress-induced defective lysosomal biogenesis.

Taken together, the evidences reported by others and our current observations suggest that NADPH Oxidase-induced oxidative stress may be the master player of defective lysosomal biogenesis and impaired autophagy (Figure 1). Therefore, Inhibition of NADPH Oxidase complex may lead us to potential therapeutic strategies for prevention of oxidative stressdependent impaired autophagy related pathological disorders.

\section{Impact of SOJ Biochemistry}

During the last decade of my research career, I am actively involved in the research areas related to Biochemistry. It is of great interest and immense pleasure to work with the intriguing mechanism(s) of actions associated with the fundamentals of Biochemistry. This includes the redox biology, oxidation, 


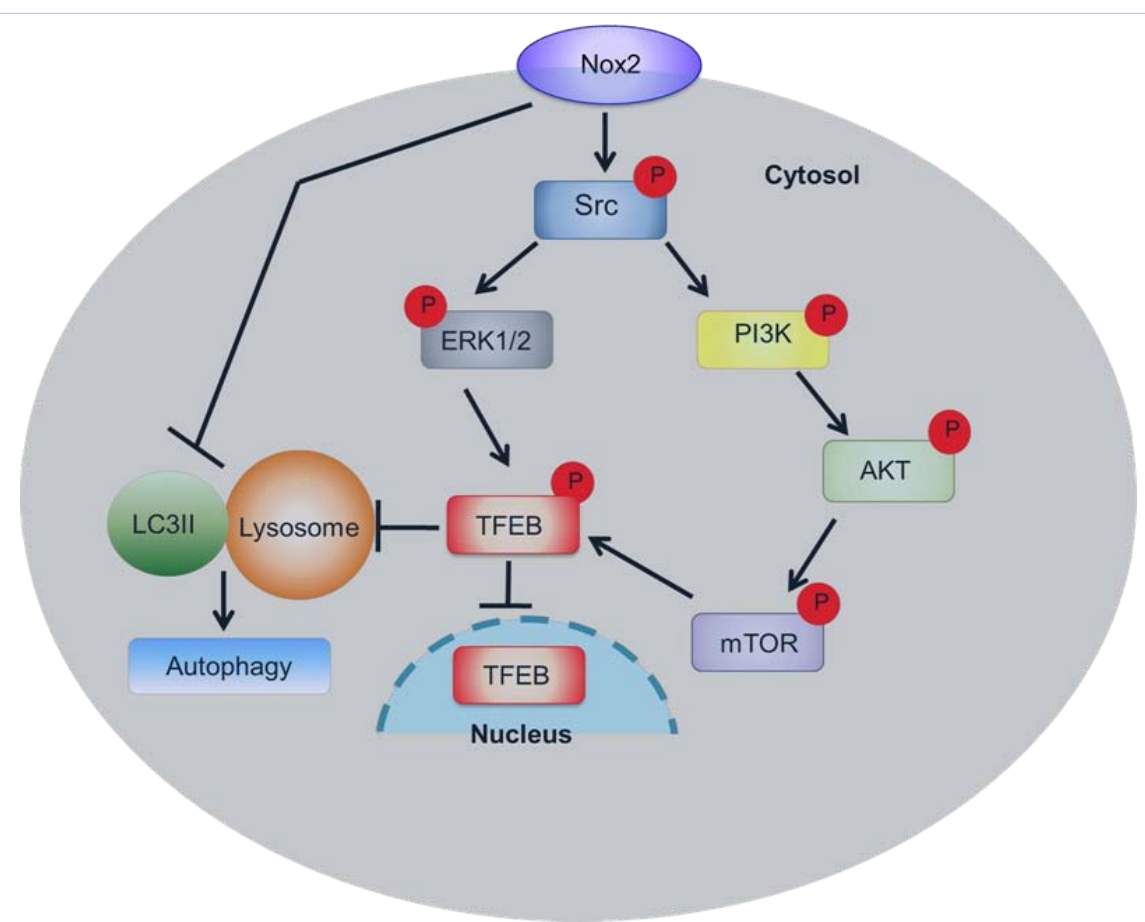

Figure 1: Proposed mechanism for NADPH oxidase (Nox2)-dependent impaired autophagy.

Autophagic machinery functions properly through autophagosome and lysosome fusion, maintaining cellular homeostasis. Activation of Nox2 depletes lysosomal biogenesis and autophago-lysosome formation

phosphorylation, protein synthesis, post-translational modification of proteins, protein folding, protein misfolding, etc. The understanding the interface between the cause and effect of myriad pathophysiological disorders involved with large number of biochemical events. Therefore, research in Biochemistry specifically got immense attention to develop novel therapeutic strategies for several pathological disorders. The knowledge gap behind the several basic biochemical reactions associated with various cellular pathways hinders the search of specific cause of many diseases such as neurodegenerative disorders, cancer pathologies, skeletal muscle disorders etc. Over the last few decades of research on Biochemistry helped us to gain better insight in to the cellular functions, which are imperative to maintain the structural and functional integrity of cells. Nevertheless, it is needless to say that we need continue putting tremendous amount of research related to Biochemistry for better understanding of the existing mechanism(s) and delve novel mechanism(s) involved with several pathological disorders. Therefore, I strongly believe that this new flexible journal (SOJ Biochemistry) will create a highly significant platform to deliver and advance the knowledge among researchers worldwide.

\section{References}

1. Rabinowitz JD and White E. Autophagy and metabolism. Science 2010; 330:1344-1348. doi: 10.1126/science.1193497.

2. Boya P, Reggiori F, Codogno P. Emerging regulation and functions of autophagy. Nat Cell Biol 2013; 15(7):713-720. doi: 10.1038/ncb2788.

3. Bampton ET, Goemans CG, Niranjan D, Mizushima N, Tolkovsky AM. The dynamics of autophagy visualized in live cells: from autophagosome formation to fusion with endo/lysosomes. Autophagy $2005 ; 1(1): 23-36$

4. Kiffin R, Bandyopadhyay U, Cuervo AM. Oxidative stress and autophagy. Antioxid Redox Signal 2006; 8(1-2):152-162.

5. Ushio-Fukai M. Localizing NADPH oxidase-derived ROS. Sci STKE 2006: 2006(349):re8.

6. Chowdhury AK, Watkins T, Parinandi NL, Saatian B, Kleinberg ME, Usatyuk PV, et al. Src-mediated tyrosine phosphorylation of p47phox in hyperoxia-induced activation of NADPH oxidase and generation of reactive oxygen species in lung endothelial cells. J Biol Chem 2005; 280(21):20700-20711.

7. Surviladze Z, Sterk RT, DeHaro SA, Ozbun MA. Cellular entry of human papillomavirus type 16 involves activation of the phosphatidylinositol 3-kinase/Akt/mTOR pathway and inhibition of autophagy. J Virol 2013; 87(5):2508-2517. doi: 10.1128/JVI.02319-12.

8. Pal R, Palmieri M, Loehr J, Li S, Abo-Zahrah R, Monroe T, et al. Srcdependent impairment of autophagy by oxidative stress in a mouse model of Duchenne muscular dystrophy. Nat Commun. 2014; 5:4425.

9. Settembre C, Di MC, Polito VA, Garcia AM, Vetrini F, Erdin S, et al. TFEB links autophagy to lysosomal biogenesis. Science 2011; 332(6036):1429-33. doi: 10.1126/science.1204592. 